\title{
The anti-inflammatory role of placental Hofbauer cells is altered in patients with chorioamnionitis: Are CYP2C8 and soluble epoxide hydrolase involved in immunomodulation?
}

\author{
Zdenek Tauber, Katerina Cizkova
}

\begin{abstract}
Aims. Hofbauer cells (HBCs) are placental macrophages playing various roles during normal and complicated pregnancies, and of the latter, chorioamnionitis is the most frequent.

Methods. In placenta with chorioamnionitis, we examined immunohistochemical expression profiles of IL-1 $\beta$, IL-10, and their potential regulators, CYP2C8 and soluble epoxide hydrolase (sEH), in Hofbauer cells and compared the results with our previously published data for normal placenta.

Results. We found that the expression profiles of the studied proteins in Hofbauer cells in chorioamnionitis differs from normal placenta. In chorioamnionitis, HBCs showed a moderate expression of IL-1 $\beta$ together with a weak expression of IL-10 and CYP2C8. Contrary to normal placenta, HBCs in chorioamnionitis express sEH. We demonstrated a moderate positive correlation between the expression of CYP2C8 and sEH in chorioamnionitis (Spearman $r=0.5654$ ), suggesting enhanced degradation of anti-inflammatory epoxyeicosatrienoic acids. Moreover, the relations of IL-1 $\beta$ and IL-10 to CYP2C8, previously described in normal placenta, disappeared. Furthermore, a weak expression of anti-inflammatory IL-10 in chorioamnionitis was accompanied by change in circularity of HBCs (Spearman $r=0.8193$ ).

Conclusion. Taken together, these findings suggest a possible alteration of the anti-inflammatory role of HBCs and its regulation in chorioamnionitis.
\end{abstract}

Key words: placental macrophages, chorioamnionitis, pro- and anti-inflammatory cytokines, CYP epoxygenases

Received: January 21, 2021; Revised: April 20, 2021; Accepted: April 21, 2021; Available online: May 11, 2021

https://doi.org/10.5507/bp.2021.026

(c) 2022 The Authors; https://creativecommons.org/licenses/by/4.0/

Department of Histology and Embryology, Faculty of Medicine and Dentistry, Palacky University Olomouc, Czech Republic Corresponding author: Katerina Cizkova, e-mail: katerina.cizkova@upol.cz

\section{INTRODUCTION}

Pregnancy is a tightly controlled immunological process in which placenta is one of the key participants ${ }^{1}$. Induction of maternal immunological tolerance to the fetus is crucial for the successful initiation and course of pregnancy. This occurs at the fetal-maternal interface very early, even before the formation of placenta itself, where the invading trophoblast comes into contact with maternal decidua containing cells of the mother's immune system $^{2}$. The fetal part of the placental immune system is represented by Hofbauer cells (HBCs), which in cooperation with the maternal immune system co-create a homeostatic and tolerogenic environment.

Hofbauer cells are macrophages of fetal origin ${ }^{3}$ localized in villous stroma, amnion and chorion leave ${ }^{4}$. HBCs are considered to be M2 macrophages ${ }^{3,5-10}$. One of their crucial functions is inducing and maintaining feto-maternal immunological tolerance ${ }^{5,11}$. Besides anti-inflammatory molecules, they are also able to produce pro-inflammatory ones, such as IL-1 $\beta$ (ref. ${ }^{12}$ ). Interestingly, the cytokine production of HBCs is changed during normal gravidity ${ }^{9,13}$. Moreover, they also play a role in angiogenesis and remodelling of the villous stroma and thereby contribute to successful development of blood vessels ${ }^{14,15}$.
The involvement of HBCs has also been studied in various pregnancy disorders, such as chorioamnionitis, type 1 diabetes mellitus, gestational diabetes, preeclampsia, villitis of unknown etiology, and others ${ }^{6,16-20}$. Chorioamnionitis is a common pregnancy complication. It is inflammation of the chorionic and amniotic membranes most frequently caused by a bacterial infection ${ }^{21}$. Chorioamnionitis increases the risk of preterm labour and is also associated with adverse neonatal outcomes including cerebral palsy and bronchopulmonary dysplasia ${ }^{22-24}$.

Production of pro- and anti-inflammatory molecules is a tightly regulated process. One group of immuno-modulating molecules is the epoxyeicosatrienoic acids (EETs). Four regioisomers of EETs, namely 5,6- 8,9-, 11,12- and 14,15-EET, are produced via cytochrome epoxygenases, such as CYP2C8, CYP2C9 and CYP2J2. They are rapidly hydrolysed to less active dihydroxyeicosatrienoic acids (DHETs) by soluble epoxide hydrolase (sEH), or they are incorporated into membrane phospholipids ${ }^{25}$. EETs, are biologically active eicosanoids with many functions in the organism. They play a role in regulation of intracellular signalling molecules, protein kinases activity, mitogenesis, cellular differentiation, regulation of ion transport, angiogenesis and promote regeneration processes ${ }^{25,26}$. Generally, EETs are considered the anti-inflammatory 
molecules ${ }^{27}$. The exact role of EETs in immuno-modulation may be dependent on EET regioisomer. Bystrom et al. confirmed that in alternatively activated macrophages (M2) 11,12-EET had an anti-inflammatory function whereas 8,9-EET regioisomer increased TNF $\alpha$ suggesting its role as a pro-inflammatory molecule ${ }^{28}$.

Our recent study, on normal human placenta, revealed the potential role of CYP2C8 in regulation of IL-10 and IL-1 $\beta$ in Hofbauer cells ${ }^{13}$. Specific information about the immunohistochemical expression of IL-10 and IL-1 $\beta$ in HBCs in placenta with chorioamnionitis is still missing. The aim of this study was to describe the IHC expression of IL-1 $\beta$, IL-10, CYP 2C8 and sEH in HBCs in placenta with chorioamnionitis, to estimate their potential relations, and thus, to extend our knowledge about the role of $\mathrm{HBCs}$ in regulation of the immune response. Moreover, we examined if the shape of $\mathrm{HBCs}$ reflects production of interleukins.

\section{MATERIAL AND METHODS}

\section{Tissue samples}

We used formalin-fixed, paraffin embedded samples of placenta with chorioamnionits $(n=19)$ obtained from archive of the Department of Clinical and Molecular Pathology, Faculty of Medicine and Dentistry, Palacky University Olomouc. Characterization of the samples is summarized in Table 1. The use of the samples was approved by the Ethics Committee of the University Hospital and the Faculty of Medicine and Dentistry, Palacky University.

\section{Immunohistochemistry}

All proteins of interest were detected in $4 \mu \mathrm{m}$ thick paraffin sections by two-step indirect immunohistochemistry. The slides were deparaffinized, hydrated and the heat antigen retrieval in citrate buffer $\mathrm{pH} 6$ was performed. Non-specific background staining was blocked by Protein Block (Dako) for $30 \mathrm{~min}$ at room temperature (RT). Then the samples were incubated with primary antibodies for $1 \mathrm{~h}$ at RT. The following primary antibodies were used: rabbit polyclonal antibodies against CYP2C8 (GeneTex; GTX113666) at dilution 1:100, IL-10 (Abcam; ab34843) at dilution 1:400 and IL-1 $\beta$ (Novus Biologicals, NBP119775 ) at dilution 1:100 and mouse monoclonal antibody against sEH (Santa Cruz, sc166916) at dilution 1:200. Appropriate dilutions of primary antibodies for immunostaining were determined by staining of positive control samples, as recommended by the manufacturer. The antibodies were diluted in Dako REAL ${ }^{\mathrm{TM}}$ Antibody Diluent (Dako). Visualization was performed by Mouse/Rabbit PolyDetector DAB HRP Brown Detection System (Bio $\mathrm{SB})$. The tissue sections were counterstained with hematoxylin. Tris buffer ( $\mathrm{pH}$ 7.6) was used for washing between the various steps. The samples were then dehydrated and cover-slipped. As a negative control, the primary antibody was substituted by Tris buffer, followed by incubation with the detection system. Positive and negative controls were present in the immunostaining of samples in order to verify the staining process.

The semi-quantitative evaluation of staining intensity, according to the strength of the signal, was graded according to the following scale: 0 for a negative tissue, 1 for a weak signal, 2 for a moderate signal and 3 for a strong

Table 1. Characterization of samples.

\begin{tabular}{cccccc}
\hline No. & Donor age & Dg. & Gestational age & Grade & Stage \\
\hline 1 & 36 & acute chorioamnionitis & 34 & 2 & 3 \\
2 & 27 & acute chorioamnionitis & 26 & 1 & 2 \\
3 & 21 & acute chorioamnionitis & 32 & 1 & 1 \\
4 & 29 & acute chorioamnionitis & 33 & 2 & 2 \\
5 & 35 & acute chorioamnionitis & 26 & 2 & 3 \\
6 & 18 & acute chorioamnionitis & 32 & 2 & 2 \\
7 & 41 & acute chorioamnionitis & 34 & 1 & 1 \\
8 & 35 & acute chorioamnionitis & 25 & 2 & 3 \\
9 & 37 & acute chorioamnionitis & 22 & 2 & 1 \\
10 & 26 & acute chorioamnionitis & 19 & 2 & 2 \\
11 & 26 & acute chorioamnionitis & 25 & 1 & 1 \\
12 & 37 & acute chorioamnionitis & 15 & 2 & 3 \\
13 & 28 & acute chorioamnionitis & 26 & 2 & 2 \\
14 & 34 & acute chorioamnionitis & 17 & 1 & 1 \\
15 & 28 & acute chorioamnionitis & 26 & 2 & 2 \\
16 & 34 & acute chorioamnionitis & 34 & 2 & 3 \\
17 & 25 & acute chorioamnionitis & 33 & 1 & 1 \\
18 & 28 & acute chorioamnionitis & 33 & 1 & 1 \\
19 & 37 & acute chorioamnionitis & 31 & 1 & 1 \\
\hline
\end{tabular}


A) IHC staining of IL-10, IL-1ß, CYP2C8 and SEH
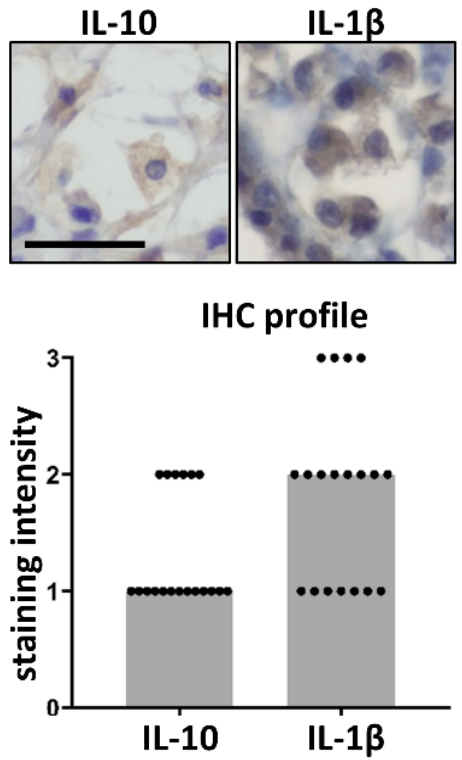

\section{CYP2C8}

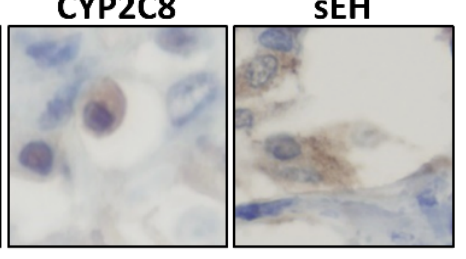

IHC profile

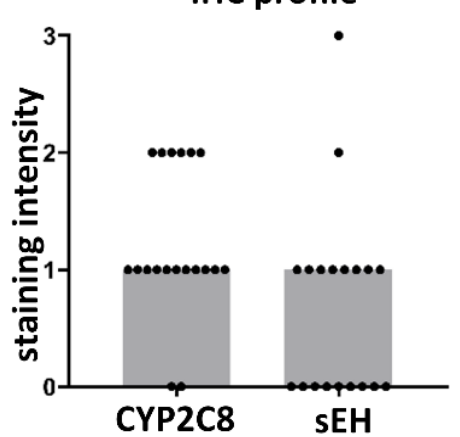

B) Correlation coefficients

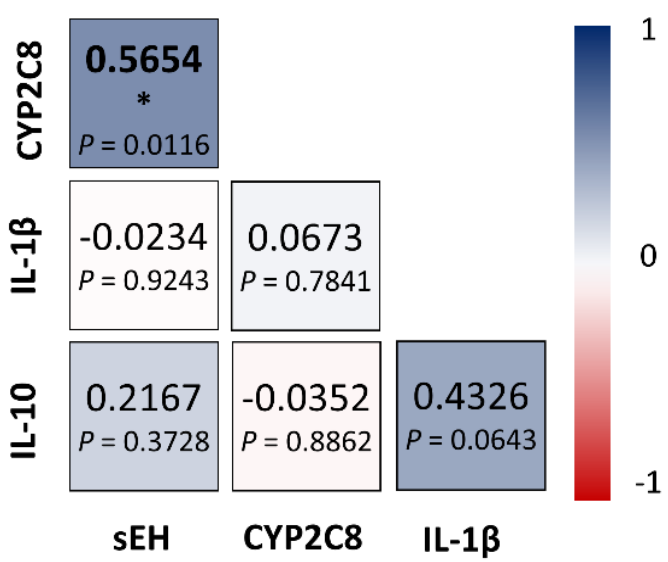

\section{C) Colocalization of CYP2C8 and CD206}
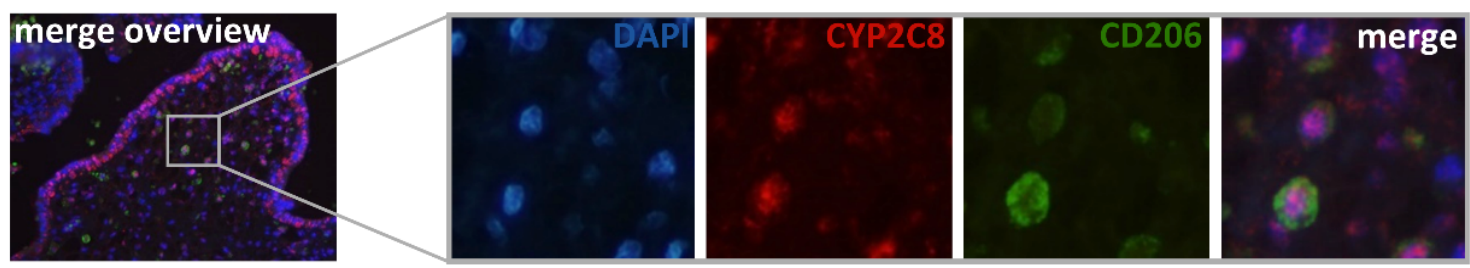

D) Colocalization of $\mathrm{SEH}$ and CD206
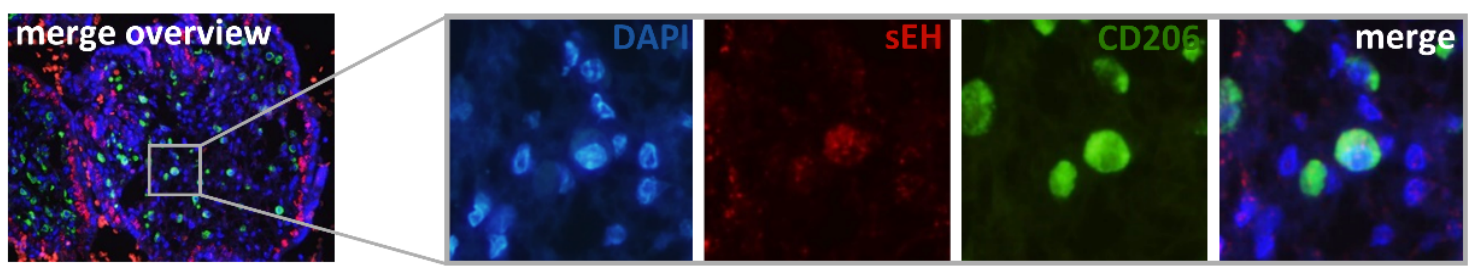

\section{E) Colocalization of sEH and CYP2C8}

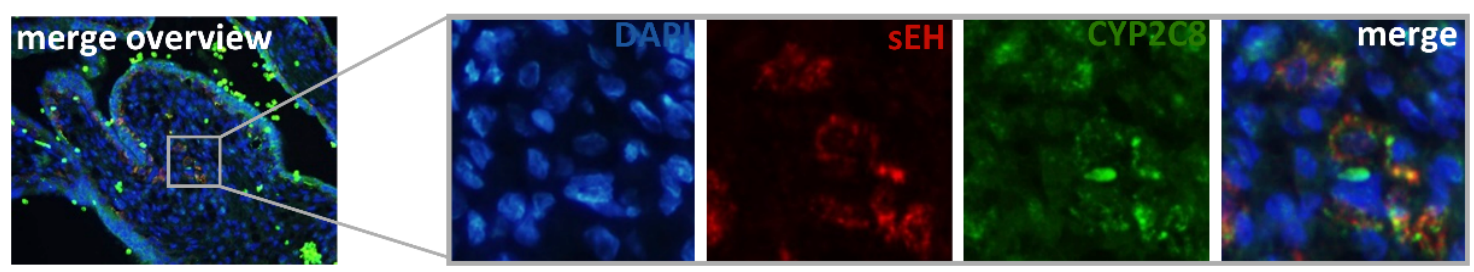

Fig. 1A. Expression of IL-10, IL-1 $\beta$, CYP2C8 and sEH in Hofbauer cells in placenta with chorioamnionitis. Microphotographs show HBCs located in stromal channels, magnification 400x, black line represents $20 \mu \mathrm{m}$. Staining intensity was evaluated as: negative (0), weak (1), moderate (2) and strong (3). Distribution of staining intensities is shown as points in the graphs (one point represents one sample; $\mathrm{n}=19$ ). Columns show medians. 1B. Spearman correlation coefficients and $P$ values, statistically significant results are marked by $* P<0.05$. 1C. Multiplex immunofluorescent staining for proof that stromal cells expressing CYP2C 8 are HBCs, detected by CD206 (marker of M2 macrophages). 1D. Multiplex immunofluorescent staining for proof that stromal cells expressing sEH are HBCs, detected by CD206 (marker of M2 macrophages). 1E. Multiplex immunofluorescent staining for proof that CYP2C8 and sEH are expressed in the same HBCs. Magnification of overview figures is 100x, details 400x. 


\section{A) Correlation coefficients}

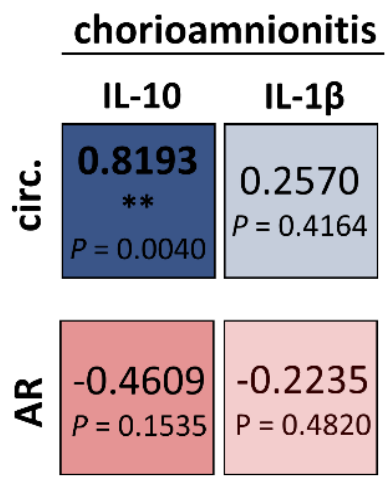

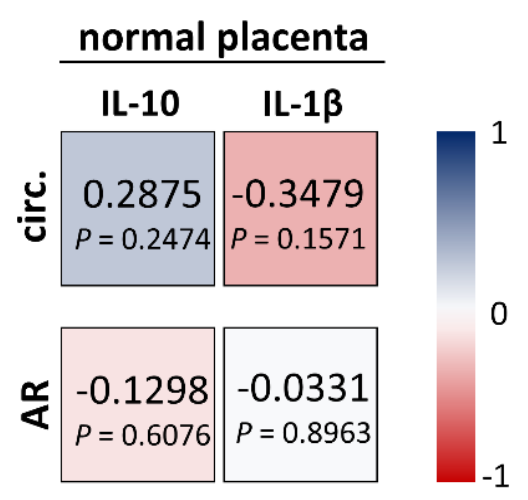

\section{B) IL-10 staining in chorioamnionitis}

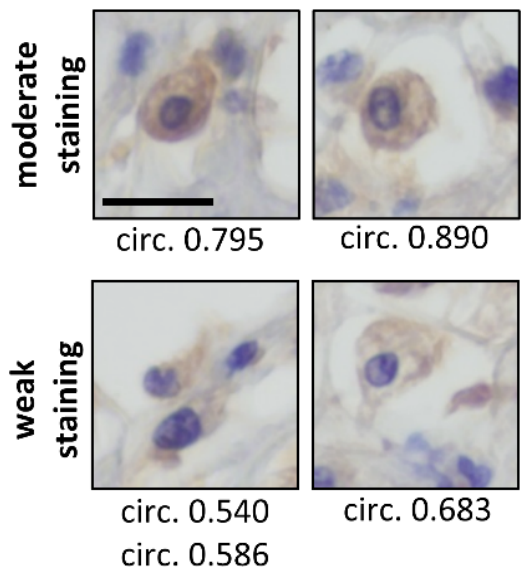

Fig. 2. Correlation of IL-10 and IL-1 $\beta$ with shape of Hofbauer cells. A) Spearman correlation coefficients for chorioamnionitis and normal placenta. Statistically significant results are marked by *. B) IL-10 staining of Hofbauer cells. Microphotographs show IL-10 staining intensity. The circularity of displayed cells is indicated directly below the microphotographs. Circularity describes the roundness of cells, where 1.0 indicates perfect circle, as the value approaches 0.0 , it indicates an increasingly elongated shape. Note higher staining intensity in rounder HBCs. Magnification 400x, black line represents $20 \mu \mathrm{m}$.

signal. The collection of samples was evaluated twice at different times. The results were summarized in graphs. The distribution of staining intensities is shown as points in the graphs (one point represents one sample) and the columns show medians. We discussed the immunohistochemical expression profiles in chorioamnionitis with our previously published data for normal placenta ${ }^{13}$.

\section{Multiplex immunostaining}

To confirm that villous stromal cells expressing CYP2C8 and sEH are Hofbauer cells, we used Opal ${ }^{\mathrm{TM}}$ 4-Color Manual IHC Kit (Perkin Elmer, NEL810001KT) according to the vendor protocol. As a marker of M2 macrophage lineage (Hofbauer cells), we used CD206 antigen. The rabbit polyclonal antibody (Abcam, ab64693) was used at dilution 1:1000. For detection of CYP2C8, and $\mathrm{sEH}$ we used the same primary antibodies as for IHC at the same dilution.

\section{Morphometric analysis}

Our recent study revealed larger variability in shape of HBCs in chorioamnionitis than in normal placenta ${ }^{10}$ thus we speculated whether changes in shapes are related to the expression of IL-10 or IL-1 $\beta$. For this analysis, we used our previously published data ${ }^{10,13}$ together with data obtained in this study. Two shape descriptors, circularity and aspect ratio measured by Image J program, were used. Circularity is defined as ( $4 \pi \mathrm{x}$ area/perimeter 2$)$. The values range from 1 to 0 , where 1 indicates perfect circle. As the value approaches to 0 , it indicates increasingly elongated shape. Aspect ratio describes the proportional relationship between the width and the length, i. e. cell's elongation. It is defined as (Major axis/Minor axis) of the best-fit ellipse for a cell. Aspect ratio is higher than 1. For a morphometric analysis, we used 18 samples of normal placenta (the 1 st trimester $n=10$ and the term $n=8$ ) and 11 samples of chorioamnionitis. The average circularity and aspect ratio were calculated for each sample and these values were correlated with IL-10 and IL-1 $\beta$ staining intensities.

\section{Statistical evaluation}

The Mann-Whitney test was used to evaluate the differences in expression of proteins of interest between the 2nd and the 3rd trimester samples. The relations between the expression of interleukins and CYP2C8 and sEH as well as interleukins and shape descriptors were evaluated by the Spearman's correlation coefficient. The level of significance $(P<0.05)$ was used for all performed tests. Statistically significant results are marked directly in graphs and figures by an asterisk $\left({ }^{*}\right)$. We used: ${ }^{*} P<0.05$, **for $P<0.01, * * *$ for $P<0.001$ and ****for $P<0.0001$. All calculations were performed with GraphPad Prism 8 software.

\section{RESULTS}

\section{Expression of CYP2C8, sEH, IL-1及 a IL-10 in chorioamnionitis}

The expression levels of CYP2C8, sEH, IL- $1 \beta$ and IL10 did not significantly differ between the second $(n=10)$ and the third $(\mathrm{n}=9)$ trimester samples (Mann-Whitney test $P=0.6481 ; P=0.5926 ; P=0.1340 ; P=0.1409$ respectively $)$. Moreover, the expression levels of CYP2C8, sEH, IL-1 $\beta$ and IL-10 did not significantly differ between samples with chorioamnionitis grade $1(n=8)$ and grade $2(n=11)$ (Mann-Whitney test with following $P$ values: $P=0.9709$ for CYP2C $8, P=0.2487$ for $\mathrm{sEH}, P=0.1619$ for IL- $1 \beta$, and $P=0.1770$ for IL-10). Based on these, all chorioamnionitis samples were evaluated all together.

Although CYP2C8 and both studied interleukins were expressed in a large number of $\mathrm{HBCs}, \mathrm{sEH}$ expression was limited to only few cells. In HBCs of placental samples 
with chorioamnionitis, we detected the weak expression of CYP2C8 and sEH. Furthermore, we demonstrated the weak expression of IL-10, along with the moderate expression of IL-1 $\beta$, see Fig. 1A. To confirm that HBCs produce both CYP2C8 and sEH we performed a colocalization of these proteins, see Fig. 1C, D, E.

\section{Correlation of CYP2C8, sEH, IL-1及 and IL-10 expression in chorioamnionitis}

We compared the expression of CYP2C8, sEH, IL-1 $\beta$ and IL-10 in HBCs to determine if there are any relations between them in the case of chorioamnionitis and in order to compare these results with our previously published data in normal placenta samples. In the case of chorioamnionitis, we found a statistically significant moderate positive correlation between CYP2C8 expression and $\mathrm{sEH}$ (Spearman $\mathrm{r}=0.5654 ; P=0.0116$ ). The relations between the other proteins studied were not statistically significant as shown in Fig. 1B.

\section{Correlation of shape characteristics (circularity and aspect ratio) with the expression of IL-1 $1 \beta$ and IL-10}

We demonstrated a statistically significant strong positive correlation between the circularity of HBCs and IL10 expression in chorioamnionitis (Spearman $\mathrm{r}=0.8193$; $P=0.004$ ) but not in normal placenta. However, we did not find any significant relations between the shape descriptors and IL-1 $\beta$ in both, normal placenta and chorioamnionitis. For results, see Fig. 2.

\section{DISCUSSION}

The aim of this study was to describe the IHC expression of IL-1 $\beta$, IL-10, CYP2C8 and sEH in HBCs in placenta with chorioamnionitis, to estimate their potential relations, and thus, to extend our knowledge about the role of HBCs in regulation of immune response. Moreover, we examined if the shape of HBCs reflects production of interleukins.

In placenta with chorioamnionitis, we demonstrated the presence of all examined proteins in HBCs. When comparing the results with our recently published data, it is clear that the intensity of their expression differs from those observed in normal placenta ${ }^{13,29}$. In chorioamnionitis, we showed a moderate expression of IL- $1 \beta$ in HBCs while IL-10 expression was weak. The weak IL-10 expression is consistent with findings of BenAmara et al., who demonstrated reduced production of IL-10 by Hofbauer cells in chorioamnionitis compared to normal controls in vitro ${ }^{16}$. In chorioamnionitis, there was no difference in expression of examined interleukins between the 2nd and the 3 rd trimester. In contrast, there were gestational age-dependent changes in IL-10 and IL-1 $\beta$ expression in normal placenta ${ }^{13}$, when in the first trimester we detected the strong IL- $1 \beta$ expression together with the weak IL-10 expression in HBCs, while term placenta samples were IL-1 $\beta$ negative and strongly IL-10 positive ${ }^{13}$. The change of IL-10 and IL- $1 \beta$ expression in HBCs during normal pregnancy is probably related to establishing immunotol- erance during the first trimester ${ }^{1}$ and promoting resolution of the inflammatory process associated with term labour $^{30}$.

It has been demonstrated that chorioamnionitis increases the risk of preterm labour ${ }^{31}$. It is well documented that pre-term birth is associated with disruption of the balance between pro- and anti-inflammatory cytokines, such as decrease in IL-10 and / or increase in IL-1 $\beta$ (ref. ${ }^{16,30,32,33}$ ). Inflammatory environment has also consequences for offsprings. Girard et al. detected the increased expression of IL- $1 \beta$ and TNF $\alpha$ in HBCs by IHC after LPS stimulation in rats. It was accompanied by both forebrain white matter and motor behavioural alterations in the offspring ${ }^{34}$. In humans, it is known that chorioamnionitis is associated with adverse neonatal outcomes including cerebral palsy and bronchopulmonary dysplasia ${ }^{22-24}$.

There is growing evidence, that arachidonic acid metabolizing enzymes, such as CYP epoxygenases play a role in immuno-modulation. In placenta with chorioamnionitis, we detected the weak expression of both, CYP2C8 and sEH. HBCs in normal placenta showed gestational age-dependent increase in CYP2C8 expression. Interestingly, $\mathrm{HBCs}$ in the case of normal pregnancy were sEH negative ${ }^{29}$. Limited data regarding IHC detection of CYP epoxygenase and sEH expression in chorioamnionitis is available. Recently Hung et al. described an increase in sEH expression in placenta samples with chorioamnionitis compared to normal ones, however, its main source was trophoblast. Furthermore, these authors described sEH positivity in some mesenchymal cells without closer specification of the cell type ${ }^{35}$. We demonstrated that $\mathrm{sEH}$ producing cells are HBCs by colocalization of sEH with CD206, marker of M2 macrophages. According to our best knowledge, this is the first evidence of this.

In the case of chorioamnionitis, there was no statistically significant relation between the expression of CYP2C8 and IL-1 $\beta$ and IL-10 as in HBCs in normal placenta $^{13}$ where we found the strong positive correlation between the expression of CYP2C8 and IL-10 together with the strong negative correlation of CYP2C8 with IL-1 $\beta$. These results suggest that CYP2C8 is one of the key enzymes for induction and stabilization of anti-inflammatory state of HBCs because of EETs synthesis ${ }^{13}$. Our results obtained in chorioamnionitis may indicate a disruption of regulatory mechanisms between the synthesis of pro- and anti-inflammatory cytokines by Hofbauer cells. Contrary to our previous results obtained for normal placenta, we demonstrated the statistically significant moderate positive correlation of CYP2C8 and sEH expression in chorioamnionitis HBCs. Furthermore, we confirmed that CYP2C8 and sEH are products of the same HBCs. Based on our results, one can speculate that lower CYP2C8 expression leads to decrease in EETs concentration which is enhanced by their hydrolysis via $\mathrm{sEH}$.

CYP epoxygenases convert arachidonic acid to four regioisomers of EETs, namely 5,6- 8,9-, 11,12- and 14,15-EET. Each CYP epoxygenase produces several regioisomers but in different ratios. The exact role of individual EET molecules remains elusive. Generally, EETs are considered the anti-inflammatory molecules acting 
via several pathways ${ }^{27}$. EETs inhibited the IL-1 $\beta$-induced activation of NF- $\mathrm{kB}$ ( ref. $^{28}$ ). The anti-inflammatory effect of EETs can be also mediated via activation of STAT3. It is known that 14,15-EET, product of CYP2C8, promote tyrosine-705 phosphorylation and nuclear translocation of STAT3 $\left(\right.$ ref. $^{36}$ ) and that the anti-inflammatory effect of IL10 in macrophages is dependent on the phosphorylation of STAT3 at the same position ${ }^{37}$. This is also supported by the results obtained by BenAmara et al., who showed that reduced production of IL-10 by Hofbauer cells in patients with chorioamnionitis compared to normal controls was associated with disrupted phosphorylation of Stat and AKT pathways ${ }^{16}$.

The exact role of EETs in immuno-modulation may be dependent on EET regioisomer as well as underlying activation state of the macrophages. Bystrom proved that in classically activated macrophages (M1), 11,12- and 8,9EETs had an anti-inflammatory function. On the other hand, in alternatively activated macrophages (M2), 11,12EET had an anti-inflammatory function whereas 8,9-EET regioisomer increased TNF $\alpha$ suggesting its role as the proinflammatory molecule ${ }^{28}$. In normal placenta, the major regulator of IL-10 and IL-1 $\beta$ expression in HBCs appears to be CYP2C8 (ref. ${ }^{13}$ ), which produces 14,15 - and 11,12EET in a comparable ratio ${ }^{38}$. Both of these regioisomers are considered to be anti-inflammatory. In chorioamnionitis, the expression of CYP2C8 is accompanied by the expression of $\mathrm{sEH}$. Because of 14,15-EET is preferable substrate for $\mathrm{sEH}\left(\right.$ ref. $\left.^{25}\right)$, the concentration of 14,15-EET is presumably low and could lead to disruption of the antiinflammatory function. Considering the fact that we have demonstrated the expression of this enzyme exclusively in chorioamnionitis samples, inhibition of sEH activity may thus represent a potential target for treatment of the disease.

Interestingly, the changes in expression profiles of studied interleukins between chorioamnionitis and normal placenta are also accompanied by the changes in HBCs shape characteristics. Our recent results showed higher variability in HBCs morphology in chorioamnionitis in comparison to normal placenta ${ }^{10}$. In chorioamnionitis, our results revealed the strong correlation between IL-10 expression and circularity of HBCs but this relation was not found in normal placenta. In accordance with our results, Eligini et al., described higher production of anti-inflammatory cytokines, including IL-10, in rounder cells than in spindle-like ones in the model of peripheral blood isolated monocyte-derived macrophages ${ }^{39}$. In contrast, Schliefsteiner et al. recently published that $\mathrm{HBCs}$ were rounder under the pro-inflammatory stimuli, while the anti-inflammatory stimulation increased the proportion of spindle-like cell ${ }^{40}$. Although several authors state that the round shape of cells represents M2 macrophages with the anti-inflammatory function, while the spindle-like shape is associated with the pro-inflammatory M1 macrophages ${ }^{39,41-43}$, it has been shown that HBCs maintained their M2 phenotype e, $^{6,10,40}$. It seems that the HBCs shape is associated with cytokine production rather than M1 or M2 phenotype.

\section{CONCLUSION}

We have shown that the expression profile of studied proteins in Hofbauer cells in chorioamnionitis differs from normal placenta. In chorioamnionitis, HBCs showed the moderate expression of IL- $1 \beta$ together with the weak expression of IL-10 and CYP2C8. In contrast to normal placenta, HBCs in chorioamnionitis express sEH. We demonstrated the relation between expression of CYP2C8 and $\mathrm{sEH}$ in chorioamnionitis, but relations between IL$1 \beta$ and IL-10 and CYP2C8, previously described by us in normal placenta, disappeared. Moreover, the weak IL-10 expression in chorioamnionitis is also accompanied by the change of HBCs shape characteristics, specifically a decrease in the circularity. Taken together, these facts suggest a possible alteration of the anti-inflammatory role of $\mathrm{HBCs}$ and its regulation in chorioamnionitis.

Acknowledgement: We thank Dr. Johana SramkovaKojecka from Department of Clinical and Molecular Pathology and Laboratory of Molecular Pathology, Faculty of Medicine and Dentistry, Palacky University Olomouc for providing chorioamnionitis tissue samples. We thank Lucie Voznakova from Department of Histology and Embryology, Faculty of Medicine and Dentistry, Palacky University Olomouc for excellent technical support.

Author contributions: Both authors contributed equally to preparing the manuscript.

Conflict of interest statement: None declared.

\section{REFERENCES}

1. Mor G, Cardenas I, Abrahams V, Guller S. Inflammation and pregnancy: the role of the immune system at the implantation site. Ann NY Acad Sci 2011;1221(1):80-7.

2. Fu B, Wei H. Decidual natural killer cells and the immune microenvironment at the maternal-fetal interface. Sci China Life Sci 2016:59(12):1224-31.

3. Kim SY, Romero R, Tarca AL, Bhatti G, Kim CJ, Lee J, Elsey A, Than NG, Chaiworapongsa T, Hassan SS, Kang GH, Kim JS. Methylome of fetal and maternal monocytes and macrophages at the feto-maternal interface. Am J Reprod Immunol 2012;68(1):8-27.

4. Bulmer JN, Johnson PM. Macrophage populations in the human placenta and amniochorion. Clin Exp Immunol 1984;57(2):393-403.

5. Böckle BC, Sölder E, Kind S, Romani N, Sepp NT. DC-sign+ CD163+ macrophages expressing hyaluronan receptor LYVE-1 are located within chorion villi of the placenta. Placenta 2008;29(2):187-92.

6. Joerink M, Rindsjö E, van Riel B, Alm J, Papadogiannakis N. Placental macrophage (Hofbauer cell) polarization is independent of maternal allergen-sensitization and presence of chorioamnionitis. Placenta 2011;32(5):380-5

7. Martinez FO, Sica A, Mantovani A, Locati M. Macrophage activation and polarization. Front Biosci 2008;13:453-61.

8. Svensson J, Jenmalm MC, Matussek A, Geffers R, Berg G, Ernerudh J. Macrophages at the fetal-maternal interface express markers of alternative activation and are induced by M-CSF and IL-10. J Immunol 2011;187(7):3671-82.

9. Swieboda D, Johnson EL, Beaver J, Haddad L, Enninga EAL, Hathcock M, Cordes S, Jean V, Lane I, Skountzou I, Chakraborty R. Baby's First Macrophage: Temporal Regulation of Hofbauer Cell Phenotype Influences Ligand-Mediated Innate Immune Responses across Gestation. J Immunol 2020; 204(9):2380-91.

10. Tauber Z, Foltynkova T, Cizkova K. Morphometric analysis of 
Hofbauer cells in normal placenta and chorioamnionitis in humans. Anat Histol Embryol 2021;50(2):396-403.

11. Yao $\mathrm{Y}, \mathrm{Xu} X \mathrm{XH}$, Jin L. Macrophage Polarization in Physiological and Pathological Pregnancy. Front Immunol 2019;10:792.

12. Reyes L, Golos TG. Hofbauer Cells: Their Role in Healthy and Complicated Pregnancy. Front Immunol 2018;9:2628.

13. Tauber Z, Chroma K, Baranova R, Cizkova K. The expression patterns of IL-1 $\beta$ and IL-10 and their relation to CYP epoxygenases in normal human placenta. Ann Anat 2021 [Epub ahead of print] doi: 10.1016/j. aanat.2020.151671

14. Seval Y, Korgun ET, Demir R. Hofbauer cells in early human placenta: possible implications in vasculogenesis and angiogenesis. Placenta 2007;28(8-9):841-5.

15. Loegl J, Hiden U, Nussbaumer E, Schliefsteiner C, Cvitic S, Lang I, Wadsack C, Huppertz B, Desoye G. Hofbauer cells of M2a, M2b and $\mathrm{M} 2 \mathrm{c}$ polarization may regulate feto-placental angiogenesis. Reproduction 2016;152(5):447-55.

16. Ben Amara A, Gorvel L, Baulan K, Derain-Court J, Buffat C, Vérollet C, Textoris J, Ghigo E, Bretelle F, Maridonneau-Parini I, Mege JL. Placental macrophages are impaired in chorioamnionitis, an infectious pathology of the placenta. J Immunol 2013;191(11):5501-14.

17. Schliefsteiner C, Peinhaupt M, Kopp S, Lögl J, Lang-Olip I, Hiden U, Heinemann A, Desoye G, Wadsack C. Human Placental Hofbauer Cells Maintain an Anti-inflammatory M2 Phenotype despite the Presence of Gestational Diabetes Mellitus. Front Immunol 2017;8:888.

18. Sisino G, Bouckenooghe T, Aurientis S, Fontaine P, Storme L, Vambergue A. Diabetes during pregnancy influences Hofbauer cells, a subtype of placental macrophages, to acquire a pro-inflammatory phenotype. Biochim Biophys Acta 2013;1832(12):1959-68.

19. Tamblyn JA, Lissauer DM, Powell R, Cox P, Kilby MD. The immunological basis of villitis of unknown etiology - review. Placenta 2013;34(10):846-55.

20. Tang Z, Buhimschi IA, Buhimschi CS, Tadesse S, Norwitz E, NivenFairchild T, Huang ST, Guller S. Decreased levels of folate receptor- $\beta$ and reduced numbers of fetal macrophages (Hofbauer cells) in placentas from pregnancies with severe pre-eclampsia. Am J Reprod Immunol 2013;70(2):104-15.

21. Mueller-Heubach E, Rubinstein DN, Schwarz SS. Histologic chorioamnionitis and preterm delivery in different patient populations. Obstet Gynecol 1990;75(4):622-6.

22. Dammann O, Leviton A. Maternal Intrauterine Infection, Cytokines, and Brain Damage in the Preterm Newborn. Pediatr Res 1997;42(1):18.

23. Gaudet LM, Smith GN. Cerebral palsy and chorioamnionitis: the inflammatory cytokine link. Obstet Gynecol Surv 2001;56(7):433-6.

24. Ramsey PS, Lieman JM, Brumfield CG, Carlo W. Chorioamnionitis increases neonatal morbidity in pregnancies complicated by preterm premature rupture of membranes. Am J Obstet Gynecol 2005;192(4):1162-6.

25. Spector AA, Fang X, Snyder GD, Weintraub NL. Epoxyeicosatrienoic acids (EETs): metabolism and biochemical function. Prog Lipid Res 2004;43(1):55-90.

26. Panigrahy D, Kalish BT, Huang S, Bielenberg DR, Le HD, Yang J, Edin ML, Lee CR, Benny O, Mudge DK, Butterfield CE, Mammoto A Mammoto T, Inceoflu B, Jenkins RL, Simpson MA, Akino T, Lih FB, Tomer KB, Ingber DE, Hammock BD, Falck JR, Manthati VL, Kaipainen A, D’Amore PA, Puder M, Zeldin DC, Kieran MW. Epoxyeicosanoids promote organ and tissue regeneration. Roc Natl Acad Sci U S A 2013;110(33):13528-33.

27. Thomson SJ, Askari A, Bishop-Bailey D. Anti-iflammatory effects of epoxyeicosatrienoic acids. Int J Vasc Med 2012;2012:605101.
28. Bystrom J, Wray JA, Sugden MC, Holness MJ, Swales KE, Warner TD, Edin ML, Zeldin DC, Gilroy DW, Bishop-Bailey D. Endogenous epoxygenases are modulators of monocyte/macrophage activity. PLoS One 2011;6(10):e26591.

29. Cizkova K, Tauber Z. Time-dependent expression pattern of cytochrome P450 epoxygenases and soluble epoxide hydrolase in normal human placenta. Acta Histochem 2018;120(6):513-9.

30. Pineda-Torres M, Flores-Espinosa P, Espejel-Nunez A, EstradaGutierrez G, Flores-Pliego A, Maida-Claros R, Zaga-Clavellina V. Evidence of an immunosuppressive effect of progesterone upon in vitro secretion of proinflammatory and prodegradative factors in a model of choriodecidual infection. BJOG 2015;122(13):1798-807.

31. Døllner H, Vatten L, Halgunset J, Rahimipoor S, Austgulen R. Histologic chorioamnionitis and umbilical serum levels of pro-inflammatory cytokines and cytokine inhibitors. BJOG 2002;109(5):534-9.

32. Chatterjee $P$, Chiasson VL, Bounds KR, Mitchell BM. Regulation of the Anti-Inflammatory Cytokines Interleukin-4 and Interleukin-10 during Pregnancy. Front Immunol 2014;5:253.

33. Puchner K, lavazzo C, Gourgiotis D, Boutsikou M, Baka S, Hassiakos D, Kouskouni E, Economou E, Malamitsi-Puchner A, Creatsas G. Midtrimester amniotic fluid interleukins (IL-1beta, IL-10 and IL-18) as possible predictors of preterm delivery. In vivo 2011;25(1):141-8.

34. Girard S, Tremblay L, Lepage M, Sébire G. IL-1 receptor antagonist protects against placental and neurodevelopmental defects induced by maternal inflammation. J Immunol 2010;184(7):3997-4005

35. Hung TH, Chen SF, Hsu JJ, Hsieh CC, Hsueh S, Hsieh TT. Tumour necrosis factor-alpha converting enzyme in human gestational tissues from pregnancies complicated by chorioamnionitis. Placenta 2006;27(9-10):996-1006.

36. Mitra R, Guo Z, Milani M, Mesaros C, Rodriguez M, Nguyen J, Luo X, Clarke D, Lamba J, Schuetz E, Donner DB, Puli N, Falck JR, Capdevila J, Gupta K, Blair IA, Potter DA. CYP3A4 mediates growth of estrogen receptor-positive breast cancer cells in part by inducing nuclear translocation of phospho-Stat3 through biosynthesis of (+/-)-14,15-epoxyeicosatrienoic acid (EET). J Biol Chem 2011;286(20):17543-59.

37. Williams L, Bradley L, Smith A, Foxwell B. Signal transducer and activator of transcription 3 is the dominant mediator of the antiinflammatory effects of IL-10 in human macrophages. J Immunol 2004;172(1):567-76.

38. Zeldin DC. Epoxygenase pathways of arachidonic acid metabolism. J Biol Chem 2001;276(39):36059-62.

39. Eligini S, Crisci M, Bono E, Songia P, Tremoli E, Colombo Gl, Colli S. Human monocyte-derived macrophages spontaneously differentiated in vitro show distinct phenotypes. J Cell Physiol 2013;228(7):1464-72.

40. Schliefsteiner C, Ibesich S, Wadsack C. Placental Hofbauer Cell Polarization Resists Inflammatory Cues In Vitro. Int J Mol Sci 2020;21(3):736.

41. Porcheray F, Viaud S, Rimaniol AC, Léone C, Samah B, DereuddreBosquet N, Dormont D, Gras G. Macrophage activation switching: an asset for the resolution of inflammation. Clin Exp Immunol 2005;142(3):481-9.

42. Vogel DY, Glim JE, Stavenuiter AW, Breur M, Heijnen P, Amor S, Dijkstra CD, Beelen RHJ. Human macrophage polarization in vitro: maturation and activation methods compared. Immunobiology 2014;219(9):695-703.

43. Tedesco S, Bolego C, Toniolo A, Nassi A, Fadini GP, Locati M, Cignarella A. Phenotypic activation and pharmacological outcomes of spontaneously differentiated human monocyte-derived macrophages. Immunobiology 2015;220(5):545-54. 